\title{
El Capitalismo Jerárquico en México y su influencia sobre la innovación desde una perspectiva de la Teoría de la Burocracia
}

\author{
Ricardo Romero Pensado a
}

Resumen - Este trabajo realiza una explicación del estado actual de la innovación de la economía mexicana por medio de una investigación que busca conectar las teorías económica y administrativa a través de la aplicación de principios y escuelas de pensamiento representativas de cada una de las disciplinas mencionadas. Surge de la idea primigenia de que existe una relación entre el tipo de capitalismo presente en México (Capitalismo Jerárquico) -el cual muestra, a través de los Grupos Económicos, particularidades observadas por la teoría burocrática de Max Webery su capacidad de innovación. Así, por medio de un análisis interdisciplinario, se encuentra una relación causal entre el capitalismo y la burocracia (es decir, entre la Economía y la Administración), temas conectados entre sí por un arreglo institucional específico en forma de un círculo no virtuoso que influye directamente en el estado actual y futuro de la innovación en una economía como la mexicana.

Palabras clave - Administración, Análisis interdisciplinario, Burocracia, Capitalismo Jerárquico, Economía, Innovación.

Abstract - The following work explains the innovation status of the Mexican economy through the search of a direct link between Economics and Management by applying representative theories and principles of the involved disciplines. The investigation arouses from the basic idea of an existing relationship between the specific type of capitalism present in Mexico (Hierarchical Capitalism) -which shows, through the performance of the Mexican Business Groups, important features defined by the Bureaucratic Theory of Max Weber- and innovation. Thus, by conducting an interdisciplinary analysis, a causal link is found between Capitalism and Bureaucracy (that is, Economics and Management) in the shape of a non-virtuous cycle derived from a specific institutional arrangement that determines the current and future scenario of innovation in Mexico.

Keywords - Bureaucracy, Hierarchical Capitalism, Economics, Innovation, Interdisciplinary analysis, Management.

\section{CÓMO CITAR \\ HOW TO CITE:}

Romero-Pensado, R. (202I). EI

Capitalismo Jerárquico en

México y su influencia sobre la

innovación desde una

perspectiva de la Teoría de la

Burocracia. Interconectando

Saberes, (12), 73-88.

https://doi.org/I0.25009/is.v0il2

.2695

Recibido: 17 de febrero de 2021

Aceptado: 4 de junio de 2021

Publicado: 20 de julio de 2021

${ }^{a}$ Universidad Iberoamericana (UIA) Puebla, México origen. E-mail: romeropensado@hotmail.com 


\section{INTRODUCCIÓN}

El presente análisis trata acerca de la realidad actual de la innovación en México como resultado del sistema económico capitalista existente en el país, desde la perspectiva de una de las tres doctrinas pertenecientes a la teoría de las organizaciones que se consideran básicas²: la Burocracia de Max Weber.

Por medio de una investigación interdisciplinaria se realiza una conexión directa entre la Economía y la Administración a través de la aplicación de teorías, principios y escuelas de pensamiento representativas de cada una de ellas.

Así, a través del análisis econométrico de la relación causal directa entre el Capitalismo Jerárquico (Economía) y la Burocracia (Administración), el trabajo encuentra que estos temas (conectados entre sí por medio de un arreglo institucional específico en forma de un círculo no virtuoso) dan una explicación sobre el nivel de innovación que existe en el país $y$, a la vez realiza, dado el vínculo investigativo de ambas disciplinas, una aportación al análisis administrativo.

La presentación del trabajo continúa de la siguiente manera: revisión de la literatura, contextualización, metodología y variables, análisis, resultados, conclusiones y referencias.

2 Marín (2006, p. 139) menciona que "a pesar de la aparente novedad de las formas administrativas, muchas de ellas han sido desarrolladas con base en el pensamiento de las doctrinas de Taylor-Fayol, Weber y Mayo".

3 Mientras el primero busca incorporar variables políticas en el análisis, el segundo abreva de la ciencia política y cuestiona los supuestos de la ortodoxia económica.

\section{REVISIÓN DE LA LITERATURA}

Se define brevemente el sustento teórico del trabajo (Capitalismo Jerárquico, Burocracia e Innovación).

\section{Capitalismo Jerárquico}

Aguirre y Lo Vuolo (2013) relatan que la añeja búsqueda académica de un modelo absoluto de sistema capitalista al que convergieran todos los países en algún momento de su historia fue cediendo su lugar a una corriente de investigación denominada Capitalismo Comparado (CC) que reconoce que existen diferentes expresiones del mismo, existiendo en dicha corriente dos enfoques bien definidos: la "Teoría de la Regulación" (TR) y las "Variedades de Capitalismo" (VC)3. El enfoque de VC se ocupa del análisis del comportamiento de las firmas y del modo en que interactúan con una particular conformación institucional.

Un trabajo pionero en esta área es el de Hall y Soskice (200I), que intenta conectar elementos del nivel micro ${ }^{4}$ con elementos a nivel macro ${ }^{5}$ en el análisis institucional de economías desarrolladas, manejando el concepto de complementariedades institucionales ${ }^{6}$ y proponiendo dos tipos de economías de acuerdo con la manera en la que las empresas resuelven sus problemas de coordinación: Economías de Mercado Liberal (Liberal Market Economies o LME) y Economías de Mercado Coordinado (Coordinated Market Economies o CME).

Sin embargo, el enfoque que se ajusta bien a las economías desarrolladas no alcanza a describir el

4 Elementos que tienen que ver con el comportamiento de actores y organizaciones.

5 Elementos que tienen que ver con la política económica y el funcionamiento institucional.

${ }^{6}$ Es decir, la manera en que tanto el desempeño como el funcionamiento de una institución afecta al desempeño y funcionamiento de otras instituciones. 
capitalismo que se observa en países menos avanzados, por lo que surge el estudio de Schneider (2009, 20I3), quien propone que existen también las Economías de Mercado de Red de Trabajo (Network Market Economies o NME) y Economías de Mercado Jerárquico (Hierarchical Market Economies o HME).

Este último autor menciona que en América Latina el capitalismo se ha caracterizado por funcionar dentro de un esquema de instituciones formales débiles, por lo que las relaciones que normalmente se darían como resultado de la coordinación o del mercado, se dan aquí a través de la jerarquía. De esta manera, en el subcontinente está presente el Capitalismo Jerárquico, en el que las organizaciones suelen tomar el papel de las instituciones, son el resultado de reglas poco claras, varían independientemente de ellas e impactan directamente a la economía, por lo que las empresas desempeñan un papel preponderante.

\section{Burocracia}

En términos generales, la burocracia ${ }^{7}$ se entiende como una organización o estructura que está caracterizada por procedimientos centralizados, división de responsabilidades, especialización del trabajo, jerarquía y relaciones impersonales.

Weber (1977, 1997, 200I) observaba en la burocracia de 1922 características que siguen presentes hoy en día ${ }^{89}$, las cuales son compartidas por las burocracias industriales y las gubernamentales, dado que

\footnotetext{
${ }^{7}$ Del francés bureaucratie (que a su vez se desprende de bureau $=$ oficina, - cratie $=$-cracia $=$ poder $\circ$ gobierno) . Huaylupo (20II, p. 289) menciona que la voz francesa bureaucratie, entendida como "el poder que emana de los empleados del Estado" fue usada por primera vez "sarcásticamente por un ministro francés del siglo XVIII" (al parecer llamado Vincent de Gourmay) durante el periodo oscuro del absolutismo monárquico.

${ }^{8}$ Una clara división del trabajo, reglas de operación bien delineadas y establecidas; una jerarquía laboral estricta; la
}

las organizaciones burocráticas tienen "importantes características en común en todos los terrenos” y tienen "sus orígenes en un grupo primario, la familia" (Berger, 1958, p. 450).

Para Weber, una vez instaurada en su plenitud, la burocracia es una de las estructuras sociales más difíciles de destruir. El burócrata individual no puede zafarse del aparato al que está unido, ya que es solamente un engrane del mecanismo que está siempre en movimiento y que le ordena ir en un sentido fijo. Además, existe una marcada tendencia en el ser humano a mantener el orden y las normas habituales, los cuales siguen vigentes independientemente de que existan en documentos que las formalicen.

\section{Innovación}

Para Drucker (2004, p. 3), la innovación es "la función específica del emprendimiento", realizada en cualquier ámbito (empresarial, público o individual). "Es el medio a través del cual el emprendedor crea nuevos recursos generadores de riqueza o dota a los recursos existentes de mayor potencial para crearla".

Gary Becker (2002), pionero en el estudio de la importancia del capital humano, fue incisivo al mencionar que la educación, la capacitación y la investigación tenían que tomarse en cuenta, además de los tradicionales tierra, trabajo y capital, como factores de producción que explicaban, por ejemplo, fenómenos complejos como el crecimiento económico. Un mayor

necesidad de una preparación específica para desempeñar un cargo; relación directa entre tamaño de la empresa y el tamaño de la burocracia que la administra.

${ }^{9}$ También se destaca una alta concentración de los recursos en manos del jefe; un estado de calculabilidad plena de los resultados económicos; un proceso de deshumanización del cargo; así como precisión, velocidad, certidumbre, conocimiento, continuidad, discreción, subordinación y reducción de costos. 
nivel de esos factores propiciaba las condiciones que generarían este último.

Marroquín y Ríos (2012) afirman que la innovación es creada en el sector de Investigación y Desarrollo (I+D) y que ello permite un crecimiento económico sostenido, siempre que haya rendimientos constantes a la innovación en términos de I+D.

Borges et al (2017), haciendo un recuento de la obra de Lundvall, mencionan que el proceso de innovación es un sistema intrincado en el que interactúa un número notable de agentes e instituciones. Por una parte, los centros de investigación (universidades, empresas e individuos) comparten conocimientos para crear nuevos productos y servicios. Por la otra, las instituciones deberán proveer un marco institucional que proteja sus derechos, provea un ambiente legal adecuado y genere la motivación para innovar.

De manera que estos últimos autores son enfáticos en mencionar y analizar el papel que el engranaje institucional (es decir, el trabajo conjunto, coordinado, consistente y duradero entre instituciones) desempeña en los procesos de innovación y en la creación de los sistemas nacionales de innovación, los cuales tienen un efecto muy importante sobre el crecimiento económico.

\section{CONTEXTUALIZACIÓN}

Se hace la ubicación contextual de las variables de estudio y la relación que guardan entre ellas.

\section{Capitalismo Jerárquico en México}

El capitalismo en México, el cual entra en la categoría de "jerárquico" propuesta por Schneider,

${ }^{10}$ Se da cuando una empresa "roba" los trabajadores calificados de otras empresas o sectores en lugar de invertir en la capacitación de sus propios trabajadores. tiene como características principales que comparte con otros países latinoamericanos: I) estar dominado por grandes grupos empresariales nacionales (GE); 2) contar con una importante presencia de compañías multinacionales (MNC); 3) tener mercados laborales atomizados y segmentados, baja densidad sindical y un considerable porcentaje de empleo informal; y 4) fuentes de financiamiento propias desarrolladas por los grupos económicos nacionales y las compañías multinacionales.

Lo anterior ocasiona que la complementariedad institucional sea en algunos casos negativa, como sucedió con el efecto expulsión que tuvo la presencia de las MNC en los sectores de alta tecnología, ya que los GE nacionales tuvieron que invertir en sectores menos intensivos en capital y con menor valor agregado a la producción y cuya inversión en $I+D$ es, comparativamente con otros países, muy baja (Schneider, 2009), como se puede ver en la Figura I.

Igualmente, en el mercado laboral se ocasiona que éste se perpetúe poco calificado: al haber una baja demanda por habilidades laborales altas, la misma inversión en el trabajo calificado disminuye por parte de las empresas y de los propios trabajadores. Incluso, se presenta el fenómeno del poaching ${ }^{10}$.Al no haber inversión en la capacitación, hay poca permanencia en el trabajo y, a la vez, las empresas se ven desalentadas a invertir en sectores de alta tecnología (Schneider, 2009). 


\section{Figura I}

Gasto en I+D como porcentaje del PIB

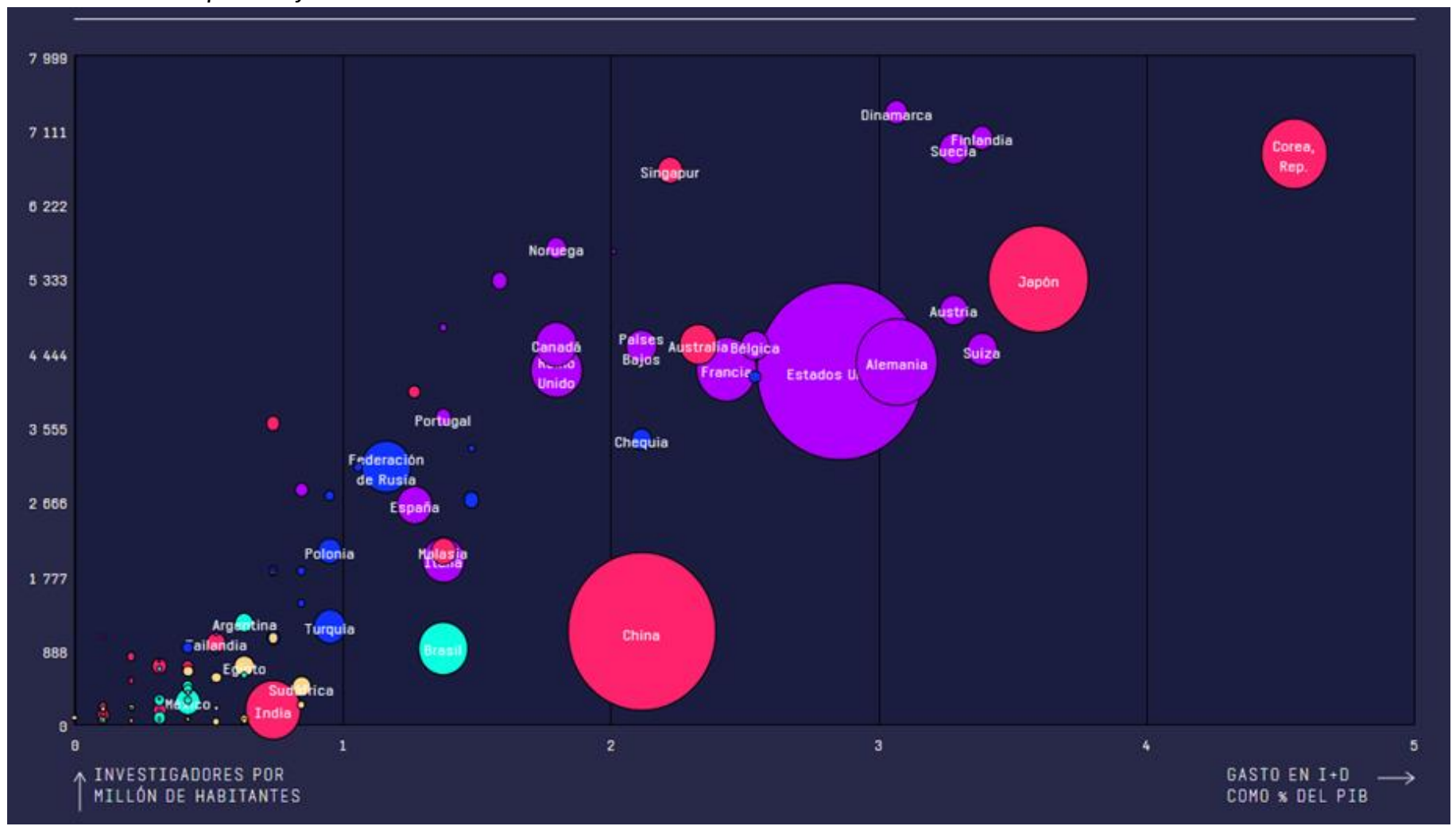

Nota. Tomado de Organización de las Naciones Unidas para la Educación, la Ciencia y la Cultura (UNESCO), 2020.

La baja capacidad de negociación colectiva, alta informalidad en sectores económicos, bajas habilidades sociales, bajo capital cultural y la inexistencia de un sistema efectivo de capacitación alimentan la "producción” de desigualdad.

Ferrando (2013) menciona que el comercio internacional actual se caracteriza por el dominio de las MNC que conservan para sí las funciones que generan más valor agregado dentro del proceso productivo (administración, comercialización y desarrollo e investigación) y fragmentan, deslocalizan y tercerizan las

II Ferrando (2013) dice que predominan dos posiciones opuestas en torno al tema: una a favor y otra en contra de la participación de los PED en las CGV. Mientras la primera ve el tema como algo que inevitablemente traerá consigo beneficios a mediano y largo plazos a los PED y pugna funciones menos valiosas (manufactura) alejándose del tradicional comercio de bienes y servicios y tendiendo hacia un comercio de "tareas".

De esta manera, existe una apropiación de valor por parte de las MNC (que generalmente pertenecen a países desarrollados) en detrimento de las empresas de los países en desarrollo (PED)"'. Así, las PYMES pertenecientes a los PED son a menudo las receptoras de las tareas más básicas dentro de la cadena global de valor (CGV).

por la profundización de la liberalización comercial, la segunda pone en duda la claridad de dichos beneficios e incluso lo ve como un instrumento de dominación y extracción de rentas y valor por parte de las MNC. 
Las Figuras 2 y 3 muestran cómo, derivado de lo anterior, se componen las exportaciones e importaciones mexicanas. México exporta principalmente (en orden de importancia por su valor) automóviles, autopartes, computadoras personales, camiones de reparto y teléfonos ${ }^{12}$. Importa (también en orden de prevalencia) petróleo refinado, autopartes, partes para máquinas de oficina, automóviles y computadoras ${ }^{13}$. Productos e insumos, todos, de la manufactura intensiva en mano de obra barata que ensambla artículos tecnológicos externos.

\section{Figura 2}

Composición de las exportaciones mexicanas en 2018

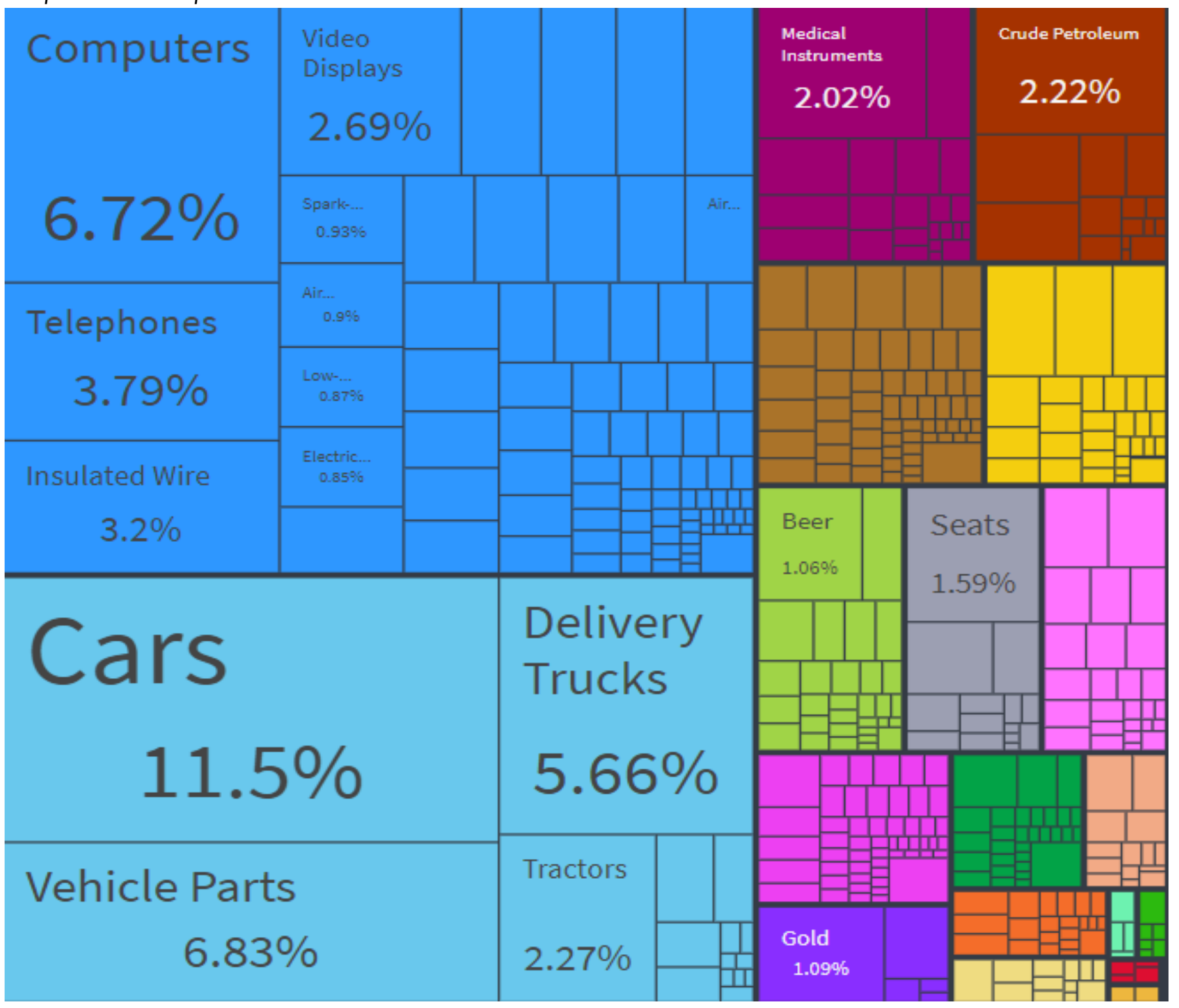

Nota. Tomado de Observatory of Economic Complexity, MIT, 2020.

${ }^{12}$ Con valores de 50.7, 30.1, 29.7, 25.0 y $16.7 \mathrm{mil}$ millones de dólares, respectivamente (OEC, 2020).
${ }^{13}$ Con valores de $31.3,27.4,16.8,10.5$ y 7.49 mil millones de dólares, respectivamente (OEC, 2020). 


\section{Figura 3}

Composición de las importaciones mexicanas en 2018

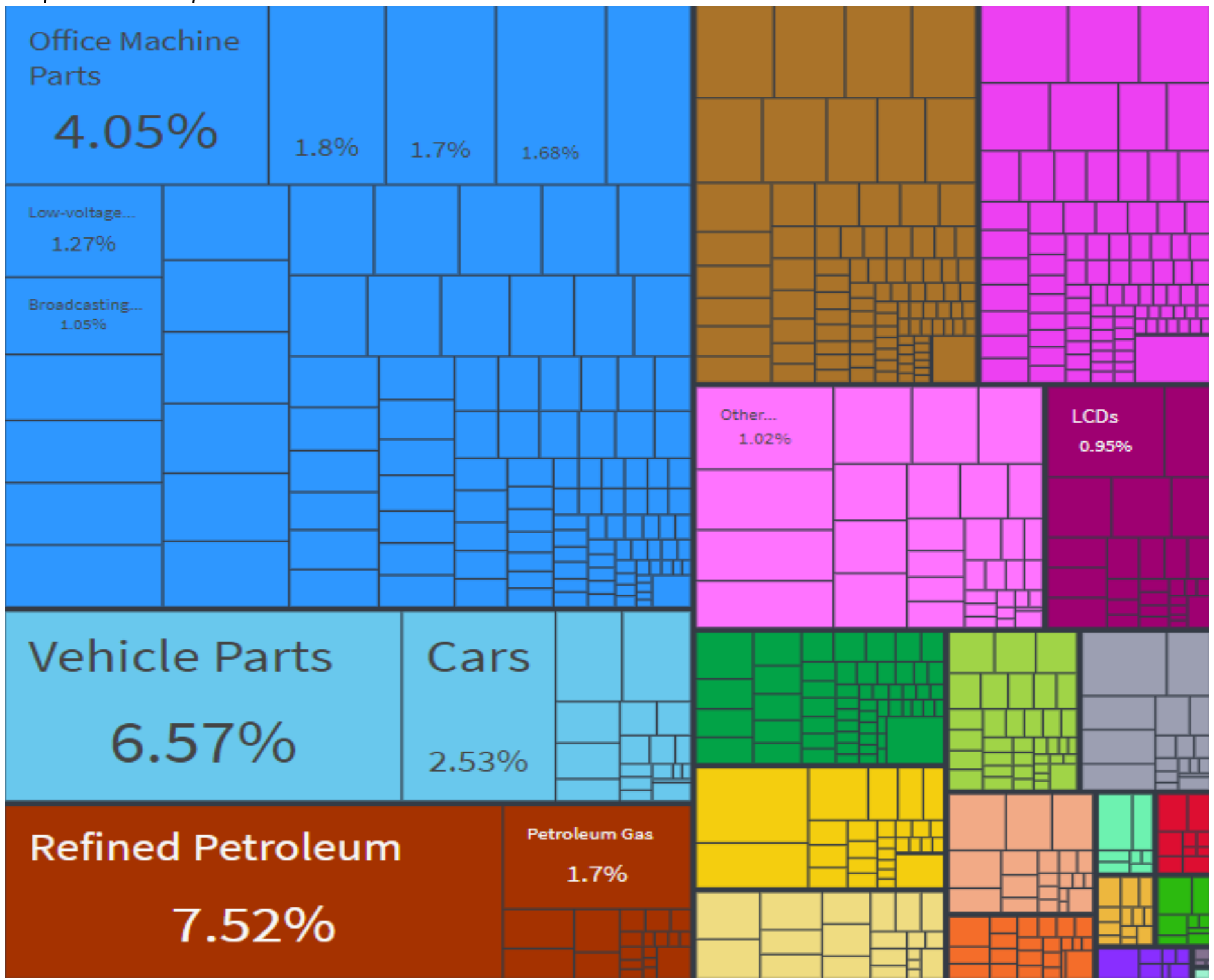

Nota. Tomado de Observatory of Economic Complexity, MIT, 2020.

En los últimos 30 años la economía mexicana pasó a estar guiada por el exterior, a pesar de mostrar una "desconexión entre la plataforma exportadora y la producción interna, así como una integración extremadamente pobre de la producción nacional con el sector externo", principalmente los Estados Unidos ${ }^{14}$.

${ }^{14}$ Bizberg ejemplifica la orientación hacia el exterior de la economía mexicana con cifras relativas al gran aumento de las exportaciones e importaciones del país a partir de los
Así, México realizó una integración dependiente o pasiva con los mercados mundiales y "complementa su dependencia de la inversión extranjera dentro de una desarticulada lógica regional de subcontratación" (Bizberg, 2014, p. 52-54).

años noventa del siglo $X X$, con una gran concentración de las exportaciones hacia EE. UU. (85\%).

\section{(cc) $\mathrm{EY}$-NC-ND}




\section{Los GE mexicanos y el Capitalismo Jerárquico}

Los GE constituyen una de las principales características del capitalismo jerárquico de México. A través de ellos y su evolución se puede entender el devenir económico del país desde prácticamente principios del siglo XX (Castañeda 2010). Los GE siguen un proceso de adaptación a las condiciones económicas, pero a la vez, dado su poder económico creciente, también influyen en ellas y en el entorno institucional en que se desenvuelven 151617181920.

En los últimos 30 años, la apertura económica ha permitido que los GE crezcan de manera impresionante, a partir de que "los procesos de neoliberalización y democratización han interactuado con

${ }^{15}$ Por ejemplo, de acuerdo con Castañeda (2007), en el periodo posrevolucionario caracterizado por los años posteriores a la Segunda Guerra Mundial, los GE nacionales obtenían precios subsidiados por parte de las paraestatales, contratos de obra pública, aprovechaban la existencia de mercados cautivos oligopólicos al tratarse de los años de economía cerrada.

${ }^{16}$ En un entorno de nacionalismo, mercados cautivos y restricciones comerciales internacionales, no hubo necesidad de hacer crecer la productividad ni de generar nuevas tecnologías, por lo que los GE no lo hicieron.

17 Otro ejemplo, de acuerdo con Castañeda (2010) es el auge petrolero. En este periodo, dicho excedente hizo posible el crédito internacional barato. Los GE, al tener su brazo financiero, accedían libremente a dichos créditos.

18 Posteriormente, la nacionalización bancaria de 1982 (que coincidió con el inicio de la crisis de la deuda externa) no afectó mucho a los GE, ya que el gobierno permitió que éstos conservaran su brazo financiero a través de las casas de bolsa. Y al llegar el momento de la privatización de las paraestatales y los bancos en los años 90 , los principales beneficiarios fueron los $\mathrm{GE}$

${ }^{19}$ Castañeda (2007) menciona que a mediados de esa década se vivió una crisis bancaria-cambiaria de grandes proporciones. Dicha crisis se convirtió en una crisis financiera y económica con repercusión mundial (el Efecto Tequila). El autor evidencia que los accionistas mayoritarios de los GE beneficiaron a sus empresas a través del crédito relacionado de sus bancos, aún a costa de la propia estabilidad bancaria, lo que provocó una incipiente crisis que, para finales de 1994 (el "error de diciembre"), fue imposible contener. un sector privado más vocal que ha visto incrementado su acceso al gobierno de manera considerable $y$ ha logrado operar de manera más autónoma y profesional” (Hogenboom, 2014, p. 296) ${ }^{21}$.

Se puede colegir, siguiendo a Alarco y del Hierro (2010), Castañeda (2007 y 2010), Chavarín (20II), Rocha (20I2) y Yiu et al (2007), que los GE, en su búsqueda legítima de beneficios, han provocado, en los diversos estadios del desarrollo económico nacional, que: i) el gobierno no obtuviera los ingresos esperados por cobro de impuestos 22 ; ii) los trabajadores perdiesen el incentivo a prepararse ${ }^{23}$; iii) el mercado de valores en México no creciera ni se profundizara; iv) se inhibiera la innovación ${ }^{24}$; v) se generara una menor competitividad ${ }^{25}$;

20 Un ejemplo más es que, a raíz de la apertura económica, los GE enfrentaron un panorama de mayor competencia, por lo que éstos dejaron atrás algunas de sus prácticas "ineficientes que velaban exclusivamente por los intereses de los accionistas con control mayoritario" (Castañeda, 2010, p. 6I4). Es decir, el abandono de prácticas ineficientes solamente se hizo cuando el mercado así lo exigió y los GE no tuvieron manera de continuar obsoletos o sobreinvirtiendo en capacidad instalada.

${ }^{21} \mathrm{Al}$ respecto, Esquivel (2015), al analizar el comportamiento de la riqueza de cuatro empresarios mexicanos (Carlos Slim, Germán Larrea, Alberto Bailleres y Ricardo Salinas), cabezas de sus respectivos GEs, menciona que su riqueza "es alrededor de un tercio del ingreso acumulado por casi 20 millones de mexicanos" (p. 19) y que todos ellos "derivan una parte significativa de su fortuna de sectores privatizados, concesionados y/o regulados por el sector público" (p.20).

22 Porque el GE movía las utilidades de una unidad del grupo a otra que tuviera un mejor trato fiscal o una mayor posibilidad de evadir impuestos.

${ }^{23}$ Al mover las utilidades o evadir impuestos y declarar menores ganancias, el GE no reporta utilidades para repartir ni para mejorar salarios y eso provoca dicho comportamiento en los empleados, que no ven retribuidos sus esfuerzos.

${ }^{24}$ En un ámbito de extracción o desvío de rentas, no es necesario innovar ni existe el proceso de "destrucción creativa. El GE internaliza el costo de su obsolescencia.

${ }^{25}$ El movimiento de recursos de una empresa del GE a otra le permitía sobreinvertir en ciertas industrias, 
y vi) se entorpeciera la inserción de las PYMES en las cadenas de valor globales ${ }^{26}$.

\section{Tabla I}

Los 10 mayores GE de México y Subsectores donde operan

\begin{tabular}{|c|c|}
\hline Familia Slim & $\begin{array}{l}\text { Telecomunicaciones, } \\
\text { construcción, comercio al por } \\
\text { menor, financiero. }\end{array}$ \\
\hline FEMSA & $\begin{array}{l}\text { Bebidas y tabaco, alimentos, } \\
\text { comercio al por menor, } \\
\text { plástico y hule. }\end{array}$ \\
\hline Alfa & $\begin{array}{l}\text { Alimentos, químicos, plástico y } \\
\text { hule, metalúrgica básica. }\end{array}$ \\
\hline Bimbo & Alimentos. \\
\hline Cemex & Cementos, minería. \\
\hline BAL & $\begin{array}{l}\text { Minería, fianzas, seguros, } \\
\text { pensiones, comercio al por } \\
\text { menor, financiero. }\end{array}$ \\
\hline Maseca-Banorte & $\begin{array}{l}\text { Alimentos, banca, bolsa, } \\
\text { seguros, fianzas, pensiones. }\end{array}$ \\
\hline México & $\begin{array}{l}\text { Minería, ferrocarril, bienes } \\
\text { muebles, construcción, } \\
\text { transporte, energía eléctrica }\end{array}$ \\
\hline Soriana & $\begin{array}{l}\text { Comercio al por menor, } \\
\text { inmobiliarios, apoyo a } \\
\text { negocios. }\end{array}$ \\
\hline Kaluz & $\begin{array}{l}\text { Químicos, plástico y hule, } \\
\text { energía eléctrica, construcción, } \\
\text { minería. }\end{array}$ \\
\hline
\end{tabular}

Nota. Tomado de Chavarín y Ríos, 2018 (p.185).

También se puede afirmar que los GE mexicanos se han adaptado a, e influido en, la estructura productiva del país, haciendo de ésta una en la que se fabrican predominantemente bienes y servicios simples que son el resultado de actividades intensivas en mano de obra, tareas rutinarias y economías de escala, que se encuentran en el principio $\circ$ fin de las cadenas

aumentando así las barreras de entrada y generando mercados oligopólicos.

${ }^{26}$ La vinculación de las MNC con los GE provoca que ésta no se haga con pequeñas y medianas empresas (ya que prevalece un ambiente de institucionalidad débil).

${ }^{27}$ Bimbo fabrica panes, Telmex proporciona servicios productivas, ya que la creación de productos complejos requiere de trabajadores capacitados, industrias relacionadas e instituciones inclusivas, características propias de economías más igualitarias (Hartmann et al, 2016). Véase el Tabla I.

\section{La burocracia como forma de organización de los GE}

De la sección anterior se sacan conclusiones y evidencias de la manera en la que los GE se han convertido en los agentes más importantes de la economía nacional en detrimento de otros actores (por ejemplo, las PYMES, las cuales no han accedido a los beneficios de la globalización y no han visto crecer sus ventas en los porcentajes descritos para los GE) y polarizando la distribución del ingreso.

Entendiendo a la organización empresarial moderna como una extensión de la "burocratización que atañe a la forma en que asume el trabajo... en un mundo de la burocracia, un mundo no sólo de reglamentos, sino uno que racionaliza hasta los propios movimientos del cuerpo del hombre" (Cousiño, 1998, p.52), se puede colegir que por medio de la adopción de un modelo económico ajeno a su esencia nacional, el Estado mexicano aceptó una criatura ajena que mantiene al país en el mismo tipo de jaula de hierro propuesta por Weber.

De esta manera es comprensible que en México predominen los grupos económicos que generan poco valor agregado para la economía nacional ${ }^{27}$ y que compartan protagonismo con empresas multinacionales

financieros, Grupo México explota minerales, Femsa fabrica refrescos, Cemex fabrica cemento y Televisa y TV Azteca, programas de televisión, por ejemplo. Ninguna crea tecnología ni conocimiento. Ninguna es pionera en investigación. de telefonía, Elektra vende electrodomésticos y servicios 
que hacen lo propio28. Las CGV se realizan para México en los niveles de menor valor agregado, lo cual sucede en detrimento del desarrollo del país.

Por medio del escaso fomento del capital social, poca densidad laboral, mínima inversión en investigación y desarrollo, apenas suficiente capacitación y educación y la consiguiente exigua innovación, el capitalismo jerárquico crea poco valor en este país $y$, por el contrario, multiplica sus aspectos más negativos: la extracción de rentas, la pérdida de humanidad y la expoliación ${ }^{29}$.

Es decir, los resultados más negativos de las prácticas recurrentes de los GE se identifican con los aspectos más negativos de la burocracia previstos por puestos, deshumanización de la actividad productiva y casi nula oportunidad de innovar. La mayor parte de las empresas pertenecientes a los GE se dedican a desarrollar o a producir bienes de escasa tecnología incorporada. Casi ninguna de ellas genera nuevas tecnologías o innovación. Weber: tareas repetitivas, una clara jerarquía de

\section{MetOdología Y VARIABLES}

El tipo de investigación realizado en este trabajo se considera como exploratorio y descriptivo, con un enfoque cuantitativo. El método es el denominado deductivo, utilizando un paradigma interpretativo ${ }^{30}$, por medio del análisis de información cuantitativa de las variables de capitalismo jerárquico de Schneider y enfoque burocrático de la teoría administrativa de Weber.

Para ello se utiliza el programa Stata, buscando determinar un modelo simple de Mínimos Cuadrados Ordinarios (MCO) -a partir de datos de series de tiempo- que describa la relación funcional $y=f(x)$ :

$$
Y \_t=\alpha+\beta \_l t X \_I t+\beta \_2 t X \_2 t
$$

donde: $Y \mathrm{t}=$ índice de complejidad económica de México; $X I t=P I B$ de EE. UU.; $X 2 t=$ número de patentes por millón de habitantes en México; $\alpha$ $=$ constante; $\beta \mathrm{it}=$ coeficientes $\mathrm{y} \mathrm{t}=$ tiempo $(\mathrm{en}$ años).

\section{El Índice de Complejidad Económica $(\text { ICE) })^{31}$}

EI ICE mide qué tan diversificada y compleja es la canasta de bienes exportados por un país, lo cual está determinado por sus conocimientos y sus capacidades productivas acumuladas. El ICE se calcula por medio de

${ }^{28}$ Las principales transnacionales arman vehículos y fabrican en territorio nacional sus autopartes. También arman televisores, lavadoras, computadoras. Todo se exporta como manufactura. Ninguna de estas empresas crea chips o software en el país. Como se observa en la Figura 14, los principales artículos exportados por México hacia EE. UU. son manufacturas y petróleo crudo. Sus principales importaciones de EE. UU. son los artículos necesarios para fabricar esas manufacturas.

29 Guerrero, López y Walton (2009) encuentran evidencia empírica de que el control de una empresa por parte de los billonarios incide en el uso de prácticas no competitivas y la obtención de privilegios.

${ }^{30}$ Que es el que incorpora el punto de vista de los participantes para entender la acción social, derivado de la interacción entre el observador y el fenómeno observado (Iglesias 2016).

31 La serie de tiempo del ICE de México de los últimos 36 años utilizada en esta investigación proviene del Observatorio de la Complejidad Económica (OEC, por sus siglas en inglés). 
dos variables: diversidad y ubicuidad del producto 32 (Hidalgo y Hausmann, 2009). A mayor valor del ICE, mayor complejidad económica ${ }^{33}$.

De acuerdo con Hartmann et al (2016) la idea subyacente del ICE es que las economías sofisticadas están diversificadas y exportan productos que tienen una ubicuidad baja (ya que pocos países diversificados fabrican estos productos sofisticados), mientras que las economías menos sofisticadas pueden producir apenas unos cuantos productos ubicuos ${ }^{34}$.

En el presente trabajo el ICE es la variable dependiente denominada ECIMX para el análisis en Stata y es la que mide el grado de innovación de la economía mexicana. Se considera como variable dependiente porque es la que está determinada por las variables de capitalismo jerárquico y burocracia. Es decir, la estructura productiva del país, condicionante de su capacidad de innovación, está influida por su modelo económico y por la manera en la que los GE se desempeñan en su día a día.

\section{Producto interno bruto (PIB) de EEUU $^{35}$}

EI PIB de un país se define como el valor monetario de los bienes y servicios finales producidos por una economía nacional en un periodo determinado (Parkin, 2009). Es el indicador macroeconómico por

${ }^{32}$ La diversidad se refiere a la variedad o número de productos que un país exporta y la ubicuidad se refiere al número de países que exportan un determinado producto.

33 Es decir, un valor positivo mayor de dicho indicador significa una mayor complejidad económica. Un valor negativo mayor significa una menor complejidad económica.

${ }^{34}$ Según el OEC (2020), en el quinquenio 2013-2017, Japón, Suiza, Alemania, Singapur y Suecia ocuparon, en ese orden, los primeros cinco lugares del ICE mundial (con ICEs de $2.309,2.243,2.075,1.865$ y 1.807 en el año 2017, excelencia de un país y permite saber si la economía de éste crece con respecto a periodos anteriores.

Para los fines de esta investigación, se considera al PIB de EE. UU. como una variable independiente (denominada GDPUSA) en el análisis porque su desempeño actúa como una variable del capitalismo jerárquico. Al estar la economía mexicana vinculada al exterior, la influencia económica de dicho país sobre México es muy grande y se considera que puede tener un valor explicativo importante en la determinación del grado de complejidad que la economía mexicana ha tenido a raíz de la adopción del capitalismo jerárquico.

\section{Patentes por millón de habitantes en México $^{36}$}

Es una variable independiente (denominada PATpc para el análisis en Stata) que puede capturar los efectos de la burocracia y, por lo tanto, puede ayudar a medirlos. Cuando la burocracia tiene mucha incidencia en la economía, hay tareas repetitivas y decisiones cupulares $\circ$ jerárquicas que dificultan $\circ$ incluso imposibilitan la innovación. Dado que las patentes son precisamente un reflejo de la inventiva, investigación y desarrollo, progreso tecnológico y grado de liderazgo científico, cuando se inhibe la innovación el número de patentes es bajo o casi nulo.

respectivamente); mientras que Sudán, Madagascar, Guinea, Bangladesh y Nigeria ocuparon los últimos cinco lugares (con ICEs de - I.457, - I.458, - I.5 I6, - I.7I4 y - I.902 en el año 20I7, respectivamente). México ocupó el lugar 21 en el periodo y tuvo un ICE de I.099 en el año 2017.

${ }^{35}$ La serie de tiempo utilizada en el análisis se obtuvo de la información del Banco Mundial (2020). El PIB de los últimos 36 años obtenido de esta fuente se encuentra expresado en precios constantes de 2010.

${ }^{36}$ La serie de tiempo de esta variable se obtuvo del Banco Mundial (2020). 


\section{Relación entre las variables}

De manera que se puede inferir la relación que existe entre la complejidad económica de un país (como medida de su grado de innovación) y el tipo de estructura productiva, laboral y hasta social derivada de su modelo económico.

Así, la evolución de las estructuras productivas, instituciones y capital humano de una economía se observa en el grado de complejidad de sus industrias, por lo que se colige que la mezcla de productos es una expresión de la mano de obra, las oportunidades de aprendizaje, el poder de negociación, la complejidad y diversidad de los productos y que las economías no diversificadas son más susceptibles a la captura económica y política.

En el caso del capitalismo jerárquico y la burocracia, se profundiza el ciclo no virtuoso mostrado en la Figura 4, que afecta negativamente a la innovación:

${ }^{37}$ I) Se hace la prueba de Dickey-Fuller para saber si las series de tiempo son estacionarias en niveles o en primeras diferencias. [Al hacer el análisis en niveles para cada variable, ésta será estacionaria si el valor del estadístico de prueba $Z(t)$ es mayor en términos reales que los valores de Dickey-Fuller a los niveles críticos de I, 5 y $10 \%]$.

2) Si la variable no es estacionaria en niveles, se procede a analizarla en primeras diferencias, aplicando el mismo criterio para establecer si es o no estacionaria.

3) Una vez comprobada su estacionariedad, se aplica la prueba de cointegración de Johansen para determinar si las

\section{Figura 4}

Círculo no virtuoso del Capitalismo Jerárquico en México

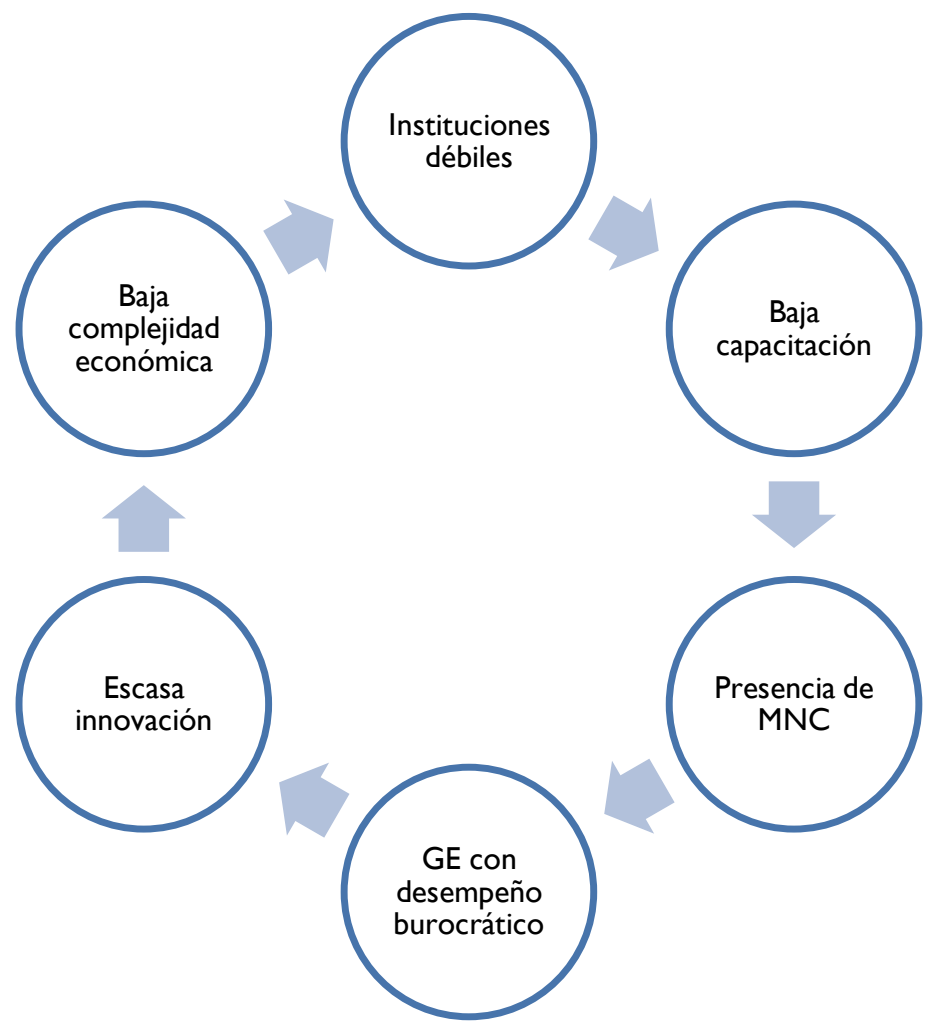

\section{ANÁLISIS}

El análisis realizado, de acuerdo con los procedimientos propuestos por Gujarati y Porter (2010) y Wooldridge (2006) constó de 4 pasos básicos ${ }^{37}$. La Tabla 2 muestra la salida de la corrida del modelo de mínimos cuadrados ordinarios (MCO) ${ }^{38}$ :

variables están o no cointegradas. En caso de comprobarse que existe la cointegración, esto significa que las variables tienen una relación de largo plazo y que ésta no es espuria.

4) Una vez verificada la cointegración de las variables, se procede a modelar por medio de MCO para conocer los signos y los coeficientes que describen la relación entre las variables. El resultado se observa en la Tabla 2.

${ }^{38}$ Se omite la presentación de las tablas de salida de Stata de cada paso por razones de espacio y porque se sobreentiende que los resultados de los pasos I a 3 están implícitos en la tabla del 4. 
Tabla 2

Resultados del modelo MCO

\begin{tabular}{|c|c|c|c|c|c|c|}
\hline \multicolumn{7}{|c|}{ regress ECIMX PATpc GDPUSA } \\
\hline \multirow[t]{2}{*}{ Source } & SS & \multicolumn{2}{|c|}{ MS } & \multicolumn{3}{|c|}{ Number of obs $=38$} \\
\hline & \multicolumn{6}{|c|}{$F(2,35)=112.90$} \\
\hline Model & 1.75991273 & \multicolumn{2}{|l|}{2.879956367} & \multicolumn{2}{|r|}{ Prob $>F$} & $=0.0000$ \\
\hline Residual & 0.272794347 & \multicolumn{2}{|l|}{35.00779412} & & R-squared & $=0.8658$ \\
\hline \multicolumn{7}{|c|}{ Adj R-squared $=0.8581$} \\
\hline Total & 2.03270708 & \multicolumn{2}{|l|}{37.05493803} & \multicolumn{2}{|r|}{ Root MSE } & $=.8828$ \\
\hline ECIMX & Coef & Std. Err. & $t \quad P>t$ & \multicolumn{3}{|c|}{ [95\% Conf. Interval] } \\
\hline PATpc & -0.0172157 & 0.0062358 & -2.76 & 0.009 & -0.0298751 & -0.0045563 \\
\hline GDPUSA & $6.17 \mathrm{e}-08$ & 4.15 e-09 & 14.84 & 0 & 5.32 e-08 & $7.01 \mathrm{e}-08$ \\
\hline cons & 0.2022539 & 0.068619 & 2.95 & 0.006 & 0.0629499 & 0.3415579 \\
\hline
\end{tabular}

Como se puede ver a partir de los resultados obtenidos por medio de Stata, las variables son estacionarias, presentan cointegración y permiten ser utilizadas para describir la relación funcional simple propuesta al principio del análisis, la cual observa un buen ajuste del modelo medido por la R2.

De acuerdo con los datos de la corrida, la relación funcional en su expresión genérica sería:

$$
Y \_t=\alpha+\beta \_I t X \_l t+\beta \_3 t X \_3 t
$$

Y de manera específica, gracias a los datos obtenidos a través de Stata, presentaría la siguiente forma:

$E C I M X=0.2022539+6.17$ e-08 GDPUSA -0.0172157 PATPC

\section{RESULTADOS}

La base teórica expuesta y analizada hasta el momento, permite entender la relación funcional encontrada:

${ }^{39}$ Esto se puede observar en la serie de tiempo de esta variable (que no se incluye por cuestiones de espacio), ya que el ICE de México ha crecido a partir de los años de
- La influencia que el comportamiento económico de EEUU descrito por su PIB tiene sobre el índice de complejidad económica de México es un estimador que responde al aspecto de capitalismo jerárquico del análisis: mediante la adopción paulatina del Capitalismo Jerárquico a través de la presencia de GE y MNC en un marco de debilidad laboral y escasa capacitación, se entienden los efectos perniciosos que dicho modelo ha tenido, en especial sobre la desigual distribución de la riqueza y el desarrollo del país, e influyendo sobre su capacidad de innovación.

- El estimador con signo positivo encontrado refleja la relación directa entre las variables. Si la economía de EE. UU. crece, la complejidad económica de México tendería a crecer también ${ }^{39}$.

- Al respecto, Cooper (1996) menciona que el aumento en la competencia derivada de

apertura económica y su menor dependencia de las exportaciones petroleras. 
relaciones comerciales más abiertas podría impulsar la innovación en los países en desarrollo. Por su parte Moncayo (2004), al analizar la teoría de la convergencia para una muestra de países, concluye que, si bien la tendencia reciente es hacia la polarización, la actividad productiva tiende a aglomerarse con una lógica de causación circular acumulativa, allí donde ya está concentrada.

- El número de patentes por millón de habitantes en México es el indicador que mide el aspecto burocrático del modelo, es decir, el que le da sentido a la parte administrativa de la investigación.

- Se considera que esta variable es el reflejo de la burocracia con la que se desempeñan los GE, dado que éstos, en su actividad económica fomentan tareas características de la burocracia (repetitivas, de escasa innovación, con poco I\&D, desalientan la educación superior, se especializan en actividades de escasa incorporación y aportación de nuevas tecnologías, ponen mayor énfasis en actividades intensivas en mano de obra, extracción de bienes primarios o en los eslabones finales de la cadena productiva con escasa aportación de valor a la misma).

- La presencia o ausencia de patentes en un país se puede considerar claramente como un indicador de su grado de innovación, modernización, uso e invención de tecnología, además de ser un reflejo de su grado de institucionalidad. De manera que el signo negativo encontrado indicaría que esta variable afecta en sentido inverso a la complejidad económica del país.

- Es decir, el grado de burocracia reflejado en su actividad económica afecta negativamente a la composición productiva del mismo, por lo que se puede considerar que esta variable incorpora el efecto tanto de la burocracia como de la presencia del capitalismo jerárquico en México, al ser los GE uno de sus actores principales.

\section{CONCLUSIONES}

El análisis econométrico realizado a través de Stata ha permitido reconocer de manera empírica la relación funcional propuesta en este trabajo, la cual permite inferir que, efectivamente, el grado de complejidad económica que tiene México, el cual es un indicador de su capacidad de innovación, se puede explicar en parte por la relación entre variables económicas y burocráticas, por lo que se puede afirmar que existe una relación de causalidad del capitalismo jerárquico y la burocracia sobre la innovación.

La variable dependiente denominada Índice de Complejidad Económica tiene poderosas implicaciones para un país, ya que determina la forma de hacer negocios que éste tiene: el país en cuestión se relaciona económicamente con otras economías nacionales de acuerdo con la cantidad de conocimiento, tecnología, calidad, abundancia y oportunidad que sus productos de exportación tienen. Un país sin valor agregado en sus productos no podrá obtener buenos términos de intercambio en el mercado internacional. Es decir, no podrá hacer negocios en el contexto de un mundo globalizado, competitivo y cambiante. 
La realidad institucional del país ha determinado que el Capitalismo Jerárquico y la Burocracia, a través de un círculo no virtuoso, perpetúen en el país sus características más negativas, las cuales han inhibido su capacidad para innovar. El modelo económico prevalente en México y el papel que los GE han desempeñado en su búsqueda de beneficios, no permitirán en el corto plazo una mejora en ese sentido.

\section{REFERENCIAS}

Aguirre, J. y Lo Vuolo, R. (20I3). Variedades de capitalismo. Una aproximación al estudio comparado del capitalismo y sus aplicaciones para América Latina. Documentos de trabajo CIEPP. (85), I-57.

Alarco, G. y Del Hierro, P. (2010). Crecimiento y concentración de los principales grupos empresariales en México. Revista CEPAL, I0I, 179-197.

Becker, G. S. (2002). The age of human capital.

Berger, M., \& Montiel, A. M. (1958). Burocracia y Desarrollo Económico. Revista Mexicana de Sociología, 20(2), 44I-45I.

Bizberg, I. (coord.) (20I4). Variedades de capitalismo en América Latina: los casos de México, Brasil, Argentina y Chile. México, D.F., México: El Colegio de México.

Borges, M., Saucedo, E. y Díaz, J. (2017). Institutional Gearing, innovation and economic growth: empirical estimation for 32 economies. DIEM: Dubrovnik International Economic Meeting, 3(I), 207-218.

Castañeda, G. (2010). 14. Evolución de los Grupos Económicos durante el periodo 1940-2008 en S. Kuntz-Ficker (Ed.), Historia económica general de México. (pp.603-634). El Colegio de México: Secretaría de Economía, México.

Castaneda, G. (2007). Business groups and internal capital markets: the recovery of the Mexican economy in the aftermath of the 1995 crisis. Industrial and Corporate Change, 16(3), 427-454.

Cooper, R. (1996). Is growth in developing countries beneficial to industrial countries? Annual World Bank Conference on development economics 1995. The World Bank. Washington, D.C.

Cousiño, C. (1998). La jaula de hierro (acerca de Max Weber). Estudios públicos, (7I).
Chavarín, R. (20I I) Los Grupos Económicos en México a partir de una tipología de arquitectura y gobierno corporativos. El Trimestre Económico, 78(309), 193-234.

Chavarin Rodriguez, R., \& Rios Almodovar, J. G. (2018). The ten largest economic groups in Mexico and their economic impact. CIMEXUS, 13(2), $175-199$.

Drucker, P. (2004). La disciplina de la innovación. Harvard business review, 82(8), 3-7.

Esquivel, G. (2015). Desigualdad extrema en México: concentración del poder económico y político. Iguales. Oxfam. https://dds.cepal.org/redesoc/publicacion?id=404 $\underline{5}$

Ferrando, A. (20/3) Las cadenas globales de valor, los países en desarrollo y sus PyMES. Instituto de Estrategia Internacional. Buenos Aires: CERA.

Guerrero, I., López Calva, L. y Waltom, M. (2009). The inequality trap and its links to low growth in Mexico, en Levi y Walton (eds.), PP. I I I-I56.

Gujarati, D. y Porter, D. (2010). Econometría. $5^{a}$ Edición. McGraw-Hill. México.

Hall, P. y Soskice, D. (200I). An introduction to varieties of capitalism, en Peter A. Hall y David Soskice, Varieties of capitalism: the institutional foundations of comparative advantage. Oxford University Press. https://scholar.harvard.edu/files/hall/files/vofcintr o.pdf

Hartmann, D., Guevara, M.R., Jara-Figueroa, C., Aristarán, M. e Hidalgo, C.A. (2016). Linking economic complexity, institutions and incomer inequality. World Development, 93, 75-93. https://www.sciencedirect.com/science/article/ab s/pii/S0305750XI5309876

Hidalgo, C. A., \& Hausmann, R. (2009). The building blocks of economic complexity. Proceedings of the national academy of sciences, 106(26), 1057010575.

Hogenboom, B. (20I4). The changing politics of lobbying: private sector organizations in Mexico. Journal of Public Affairs, 14(3-4), 296-309.

Huaylupo, J. A. (20I I). La burocracia y sus críticos en la desregulación del quehacer estatal.

Iglesias, M. (2016). Metodología de la investigación científica; diseño y elaboración de protocolos y proyectos. Buenos Aires: Noveduc.

Marín, D. (2006). El sujeto humano en la administración: una mirada crítica. Cuadernos de Administración. 19(32), 135-I56. 
Marroquín Arreola, J., \& Ríos Bolivar, H. (2012). Inversión en investigación y crecimiento económico: un análisis empírico desde la perspectiva de los modelos de I+ D. Investigación económica, $7 \mathrm{I}$ (282), I5-33.

Moncayo, E. (2004). El debate sobre la convergencia económica internacional e interregional: enfoques teóricos y evidencia empírica. eure, 30(90), 7-26.

Observatory of Economic Complexity. (2020). Explore the latest trade data. MIT. https://atlas.media.mit.edu/en/

Organización de las Naciones Unidas para la Educación, la Ciencia y la Cultura [UNESCO]. (2020). ¿Cuánto invierte su país en I+D? Instituto de Estadística.

Parkin, M., Loría, E. (2009). Macroeconomía: Versión para Latinoamérica. México, D.F.: Pearson Educación.

Rocha, J. M. (2012). Business groups as hierarchical clique structures: A conceptual and methodological discussion as it applies to the Mexican experience. British Journal of Management, 23(3), 29I-306.

Schneider, B. (2009). Hierarchical market economies and varieties of capitalism in Latin America. United Kingdom: Cambridge University Press.

Schneider, B. (20I3). Hierarchical capitalism in Latin America. Business, labor and the challenges of equitable development. New York, USA:

Cambridge University Press.

Weber, M. (200l). La ética protestante y el "espíritu" del capitalismo. Madrid: Alianza Editorial.

Weber, M. (1997). Economía y sociedad. México, D.F. Fondo de Cultura Económica.

Weber, M. (1977). ¿Qué es la burocracia? Libros Tauro. Buenos Aires. https://ucema.edu.ar/ ame/Weber_burocracia.pd $\underline{f}$

Wooldridge, J. M. (2006). Introducción a la econometría: un enfoque moderno. Editorial Paraninfo.

World Bank (2020). Patent applications, nonresidents $1980-2019$.

https://data.worldbank.org/indicator/IP.PAT.NRE S?\%20locations=MX

Yiu, D. W., Lu, Y., Bruton, G. D., y Hoskisson, R. E. (2007). Business groups: An integrated model to focus future research. Journal of Management Studies, 44(8), I55 I- 1579. 